to any conformal map of a sphere upon a plane lead to new characterizations of the Mercator, stereographic, and the general Lambert conical projections. Thus the only conformal map with straight scale curves is the Mercator; and the only circular cases are the stereographic and Lambert maps. (Received January 25, 1944.)

\title{
Statistics and Probability
}

\section{Benjamin Epstein and C. W. Churchman: On the statistics of sensitivity data.}

"Sensitivity data" is a general term for that type of experimental data for which the measurement at any point in the scale destroys the sample. The paper is a generalization of a method of treating such data due to Spearman. (C. Spearman, The method of "right and wrong cases" (constant stimuli) without Gauss' formulae, British Journal of Psychology vol. 2 (1908) pp. 227-242.) Formulae for the moments and their standard sampling errors are given. Certain minimization problems are also discussed. (Received January 26, 1944.)

\section{E. J. Gumbel: The observed return period.}

The theoretical return period $T(x)$ of a value equal to, or greater than, $x$ is defined as the inverse of the probability $1-F(x)$. The question is how to calculate, for $n$ observations, the return period $T\left(x_{m}\right)$ of the $m$ th observation $x_{m}(m=1,2, \cdots, n)$, and especially $T\left(x_{n}\right)$ of the largest observation $x_{n}$ for an unlimited variate. This problem is important in probability papers where the variate is plotted as a function of the return period. Engineers use a compromise between the exceedance interval ' $T\left(x_{m}\right)$ $=n /(n-m)$ and the recurrence interval $" T\left(x_{m}\right)=n /(n-m+1)$, namely $T\left(x_{m}\right)$ $=n /(n-m+1 / 2)$. In this case $T\left(x_{n}\right)=2 n$. If, however, the probability $F\left(x_{n}\right)$ of the median $\ddot{x}_{n}$ of the largest value is attributed to $x_{n}, T\left(x_{n}\right)=1.44 n+1 / 2$. Both methods can hardly be justified. The author attributes the probability $F\left(\tilde{x}_{n}\right)$ of the most probable largest value $\tilde{x}_{n}$ to $x_{n}$. Then $T\left(x_{n}\right)$, as is to be expected, converges toward $n$, and equals $n$ for the exponential distribution, and $n+1$ for the logistic distribution. In the same way, the probability $F\left(\tilde{x}_{1}\right)$ of the most probable smallest value $\tilde{x}_{1}$ is used, for an unlimited variate, as frequency of the smallest observation $x_{1}$. The frequencies $F\left(\hat{x}_{m}\right)$ of the intermediate $n-2$ observations are obtained by linear interpolation between $F\left(\tilde{x}_{1}\right)$ and $F\left(\tilde{x}_{n}\right)$. Thus the return periods may be determined for all observations. (Received January $27,1944$. )

\section{H. E. Robbins: On the measure of a random set.}

Let $X$, a measurable subset of Euclidean $n$-dimensional space $E$, be a random variable (for example, $X$ may be the set-theoretical sum of $N$ possibly overlapping and independently chosen unit intervals on a line with a given probability distribution for their centers). Let $m(X)$ denote the measure of $X$, and for any point $x$ of $E$ let $p(x)$ denote the probability that $X$ contain $x$. Then under very general hypotheses on $X$ it is shown that the expected value of $m(X)$ is equal to the integral over $E$ of $p(x)$. More generally, the expected value of the $r$ th power of $m(X)$ is equal to the integral over $r n$-dimensional space of the function $p\left(x_{1}, \cdots, x_{r}\right)=$ probability that $X$ contain all the points $x_{1}, \cdots, x_{r}$. (Received January $28,1944$. .) 